Annuaire suisse de politique de développement

\title{
Les fédérations cantonales de coopération au développement : une forme originale de renforcement du mouvement associatif
}

Daniel Fino

\section{(2) OpenEdition \\ Journals}

Édition électronique

URL : http://journals.openedition.org/aspd/454

DOI : $10.4000 /$ aspd. 454

ISSN : 1663-9669

Éditeur

Institut de hautes études internationales et du développement

Édition imprimée

Date de publication : 1 novembre 2004

Pagination : 99-108

ISSN : 1660-5934

\section{Référence électronique}

Daniel Fino, «Les fédérations cantonales de coopération au développement : une forme originale de renforcement du mouvement associatif », Annuaire suisse de politique de développement [En ligne], 23-2 | 2004, mis en ligne le 10 mars 2010, consulté le 07 septembre 2020. URL : http:// journals.openedition.org/aspd/454; DOI : https://doi.org/10.4000/aspd.454 


\section{Les fédérations cantonales de coopération au développement: une forme originale de renforcement du mouvement associatif}

\section{Daniel Fino*}

e but de cet article est de présenter la spécificité du type de regroupement, au niveau cantonal, d'organisations non gouvernementales (ONG) de coopération au développement. Après la présentation du cadre général, nous développerons les cinq axes de réflexion suivants, qui démontrent les avantages, mais aussi quelques questionnements liés à ce type de regroupement:

1. un enrichissement pour les associations membres;

2. une nouvelle force dans la formation de l'opinion publique;

3. la mobilisation de ressources financières publiques en faveur des ONG;

4. un mouvement d'associations qui se professionnalise;

5. pouvoir public et ONG : une relation en évolution.

Le cadre général

La façon dont les ONG de développement s'organisent dans les différents pays industrialisés pour renforcer leur position dans la société, notamment en tant qu'interlocuteurs des pouvoirs publics ${ }^{1}$, est très variée et dépend largement du système et de la culture politiques de chaque pays ${ }^{2}$. En Suisse même, on peut constater des différences entre la Suisse alémanique et la Suisse romande et latine. Les ONG de Suisse alémanique, bien qu'elles collaborent sur des thématiques spécifiques sous forme de plates-formes ou de communautés ${ }^{3}$, ne sont pas regroupées au niveau cantonal. Certaines d'entre elles ont une vocation nationale. En Suisse romande et latine, les ONG ont nettement tendance à se regrouper et une consolidation des fédérations cantonales est en cours.

Ce mouvement a commencé avec la création de la Fédération genevoise de coopération (FGC) voici déjà plus de trente ans. La FGC est demeurée longtemps la seule dans son genre jusqu'à ce que, il y a maintenant une décennie, les ONG d'autres cantons suivent l'exemple. Aujourd'hui, les fédérations sont actives dans cinq autres cantons: Vaud, Jura, Valais, Tessin et Fribourg (voir

* Chargé de cours et directeur adjoint, iuéd. Depuis quatre ans, D. Fino réalise des sessions de formation à l'intention des associations membres des fédérations cantonales (mandat DDC).

1 Pour le cas suisse, le pouvoir public est représenté par l'administration fédérale (notamment la Direction du développement et de la coopération/DDC), les cantons et les communes.

2 Dans la plupart des pays européens, les ONG font des démarches individuelles auprès de l'administration, aux niveaux tant national que régional et local.

3 La plus connue est la Communauté de travail des œuvres suisses d'entraide, qui réunit Swissaid, Helvetas, Action de carême, Pain pour le prochain, Caritas et Eper. 
tableau 1). Les motivations à la base de la création et de la consolidation des fédérations ne sont pas forcément identiques dans les six cas et chacune des fédérations a ses spécificités, compte tenu de la constellation de ses membres et des expériences vécues dans chaque canton.

Tableau 1: Vue d'ensemble des fédérations en 2003

\begin{tabular}{|c|c|c|c|c|c|c|c|}
\hline \multirow{2}{*}{$\begin{array}{l}\text { Fédérations } \\
\text { cantonales }\end{array}$} & \multirow{2}{*}{$\begin{array}{l}\text { Année de } \\
\text { création }\end{array}$} & \multirow{2}{*}{$\begin{array}{l}\text { Nbre d'associa- } \\
\text { tions membres }\end{array}$} & \multicolumn{3}{|c|}{ Principales fonctions } & \multirow[b]{2}{*}{$\begin{array}{l}\text { Projets étudiés } \\
\text { et volume de } \\
\text { financement }\end{array}$} & \multirow{2}{*}{ Partenaires } \\
\hline & & & $\begin{array}{l}\text { Appui- } \\
\text { conseil }\end{array}$ & $\begin{array}{l}\text { Information } \\
\text { lobbying }\end{array}$ & $\begin{array}{l}\text { Activités } \\
\text { culturelles }\end{array}$ & & \\
\hline Fribourg & 2003 & 5 & $x$ & $x$ & $x$ & $\begin{array}{l}\text { Pas de rôle } \\
\text { d'intermédiation } \\
\text { financière directe }\end{array}$ & \\
\hline Genève & 1966 & 47 & $x$ & $x$ & $x$ & $\begin{array}{l}47 \text { projets } \\
\text { approuvés par } \\
\text { année, volume } \\
\text { de } 8,3 \text { mio de fr. }\end{array}$ & $\begin{array}{l}\text { Contrats avec la } \\
\text { DDC, I'Etat et la } \\
\text { Ville de Genève } \\
\text { collaboration } \\
\text { avec env. } 20 \\
\text { communes du } \\
\text { Canton de Genève }\end{array}$ \\
\hline Jura & 1994 & 18 & $x$ & $x$ & $x$ & $\begin{array}{l}\text { Pas de rôle } \\
\text { d'intermédiation } \\
\text { financière directe }\end{array}$ & Canton, DDC \\
\hline Tessin & 1999 & 69 & $x$ & $x$ & $x$ & $\begin{array}{l}\text { Pas de rôle } \\
\text { d'intermédiation } \\
\text { financière directe }\end{array}$ & $\begin{array}{l}\text { Canton, DDC, } \\
\text { communes }\end{array}$ \\
\hline Valais & 1996 & 26 & $x$ & $x$ & $x$ & $\begin{array}{l}\text { Pas de rôle } \\
\text { d'intermédiation } \\
\text { financière directe } \\
\text { (introduction en } \\
\text { 2004) }\end{array}$ & $\begin{array}{l}\text { Canton, DDC, } \\
\text { communes }\end{array}$ \\
\hline Vaud & 1989 & 34 & $x$ & $x$ & $x$ & $\begin{array}{l}62 \text { projets en } \\
\text { cours de finan- } \\
\text { cement, volume } \\
\text { de } 1,2 \text { mio. fr }\end{array}$ & $\begin{array}{l}\text { Canton, DDC } \\
\text { (crédit-cadre), } \\
\text { communes }\end{array}$ \\
\hline
\end{tabular}

Pour certaines, le fait que le regroupement d'associations sous forme d'une fédération facilite l'accès à des fonds publics qui viennent compléter les fonds propres a constitué une motivation très importante. Cet intérêt a rencontré une écoute favorable de la part de certaines instances publiques - tels les cantons et des communes de Genève et de Vaud - désireuses de renforcer les activités de coopération par le biais des ONG organisées au niveau cantonal sans devoir créer une administration au niveau interne. L'interlocuteur fédéral, la DDC, qui attache une grande importance à la diversification des actions de coopération entre acteurs publics et associatifs, favorise la création de fédérations. Celles-ci permettent en effet à la DDC de développer des relations efficaces avec le monde des ONG, tant sur le plan des échanges que sur le plan des contributions financières.

Pour d'autres fédérations, les motivations suivantes étaient plus prépondérantes que le «captage» de nouveaux fonds: le désir de sortir de l'isolement des organisations de développement, de favoriser les échanges et de partager les expériences entre elles (forte motivation au Jura) ou encore de donner plus de poids à la diffusion de questions liées au développement, mais aussi de «profession- 
naliser $»^{4}$ le travail important effectué par une multitude de petites ONG (forte motivation au Tessin).

Quoi qu'il en soit, les raisons et intérêts qui déterminent la constitution des fédérations abondent ${ }^{5}$, et notre propos n'est pas de faire une comparaison entre ces motivations fédération par fédération, mais d'exposer ci-après cinq axes de réflexion qui caractérisent le paysage de la coopération au développement là où des fédérations sont présentes.

Dans aucun des cas observés on ne peut constater une volonté de «monopoliser» le champ de la coopération au développement et d'exclure, par exemple, l'accès des ONG non membres des fédérations aux financements publics. Les fédérations doivent néanmoins être conscientes de ce risque car, sans qu'elle le veuille, une fédération constitue une référence par le simple fait qu'elle demande le respect de certains critères et principes et assure un contrôle de qualité des dossiers avant d'accéder aux financements. Les ONG qui ne peuvent pas bénéficier de ce «label » peuvent être, par la force des choses, désavantagées vis-à-vis de certains bailleurs de fonds.

\section{Un enrichissement pour les associations membres}

Les fédérations favorisent les échanges d'expériences entre les associations membres. Pour certaines fédérations, cette fonction est très importante. Les associations membres, tout en gardant leur autonomie, leur indépendance et leur spécificité - elles peuvent même être parfois concurrentes -, trouvent un lieu de rencontre pour approfondir des questions communes. Prenons des thèmes comme le partenariat, l'éthique dans la coopération, l'articulation entre l'aide humanitaire et l'aide au développement, les outils de gestion ou encore l'information du public: ils représentent tous un certain nombre de problèmes rencontrés par la plupart des associations, dont personne n'a trouvé la solution idéale et qui peuvent lourdement handicaper le travail sur le terrain. La fédération est le lieu où les échanges sur ces préoccupations peuvent se dérouler en toute confiance, sans que l'échec ou les faiblesses soient exploités en défaveur de celui qui les expose, mais où tout au contraire l'apprentissage par l'erreur peut être formatif.

En effet, le besoin d'échange et la disponibilité au partage sont les signes d'une certaine «maturation» d'une association : celle-ci est ouverte à la discussion sur son expérience, elle ne craint pas la critique, elle est prête à mettre son approche en question, etc. Cette attitude de transparence et d'esprit critique n'est pas donnée automatiquement; il est parfois nécessaire d'accompagner le processus. C'est ici que les fédérations ont un rôle à jouer: faciliter le processus d'échange, l'organiser et le mener à bon terme pour en tirer des leçons profitables à l'ensemble des membres.

4 «Professionnaliser» est compris ici dans le sens d'acquérir de nouvelles compétences et de nouveaux savoirs, et non pas dans le sens d'un abandon du bénévolat.

5 Depuis deux ans, les différentes fédérations se rencontrent régulièrement pour échanger leurs expériences et discuter des questions qu'elles partagent. 


\section{Fédération jurassienne de coopération et de développement, <www.fjcd.ch>}

Comment réunir un maximum d'ONG jurassiennes, faire connaître leur travail, sensibiliser le public, les jeunes en particulier, aux questions liées au développement et à la solidarité, attirer l'attention des représentants des pouvoirs publics, etc.?

Fondée en 1994, la Fédération jurassienne de coopération et de développement (FJCD) compte à ce jour 18 associations. En automne 2000, la FJCD prend l'initiative de rassembler toutes les associations d'entraide du Jura autour d'un grand projet commun: une Fête de la solidarité. Une vingtaine d'associations répondent à l'appel et sollicitent le Collège de Delémont, qui accepte de participer à cette démarche... Le projet prend alors la forme d'une collaboration entre les associations d'entraide jurassiennes et les enseignants et élèves du Collège de Delémont. De multiples activités festives sont prévues à l'intérieur et autour de l'école. La préparation de la fête engendre de nombreux échanges entre les différents partenaires: des groupes d'animation se forment, des travaux s'engagent dans les classes, les associations rencontrent les élèves et présentent leurs projets, et toute une palette d'activités se met en place en vue de la préparation de la fête.

Ces différents travaux conduisent à une réflexion sur la problématique Nord-Sud, sur la culture des pays en voie de développement, sur le travail des associations, ici et dans les pays défavorisés, sur la solidarité en général. Ils induisent une ouverture à l'autre et au monde, et réalisent déjà plusieurs objectifs de la Fête de la solidarité, bien avant qu'elle n'ait débuté.

Isabelle Boegli Milani

Coordinatrice de la FJCD, <info@fjcd.ch>

\section{Une nouvelle force dans l'opinion publique}

Les fédérations, qui parlent au nom de toutes leurs associations membres, représentent indéniablement un poids plus important que chaque organisation individuelle. Les thématiques générales qui concernent l'ensemble de la communauté des ONG ont plus de poids quand une fédération les diffuse. Cela vaut notamment pour des thématiques relativement difficiles à faire passer et qui touchent à des questions philosophiques et éthiques, comme la solidarité internationale, le respect d'autres cultures, les raisons structurelles des situations de pauvreté, les problèmes de cohérence dans nos politiques de développement, etc. Les fédérations remplissent alors un rôle important dans la constitution de l'opinion publique, car elles sont des messagers de valeurs et de visions du monde qui s'opposent à l'injustice, à la haine et à la guerre, et qui défendent en quelque sorte le «droit au développement».

Cependant, il n'est pas toujours très facile, au niveau interne, de parvenir à un consensus entre les associations membres quand il s'agit de prendre position sur tel ou tel sujet. Chaque fédération est donc obligée de fixer les règles du jeu. Une prise de position nécessite en règle générale l'unanimité des associations (pour la FGC, la majorité suffit), sous peine de désintégration, de démissions de membres ou d'éclatement. Chaque fédération doit être très attentive à ce danger, sans pour autant qu'il lui faille renoncer à jouer ce rôle d'opinion maker. Le débat interne pour arriver à une position commune constitue le côté très positif de ce processus.

Les fédérations renforcent la situation de petites organisations dont les membres s'engagent bénévolement pour un travail de coopération au développement et elles leur donnent une voie qu'elles n'auraient pas en travaillant hors réseau. 


\section{Valais Solidaire, <www.valaissolidaire.ch $>$}

Valais Solidaire, fédération valaisanne des ONG de coopération fondée en 1996, poursuit plusieurs objectifs, comme la capitalisation des expériences, la mise en réseau des compétences, la reconnaissance comme centre de référence, l'établissement et le resserrement de liens entre le Valais alémanique et le Valais romand.

Pour se faire mieux connaître, Valais Solidaire a participé à l'Année internationale de la montagne 2002 avec la réalisation d'un projet visuel de sensibilisation, comprenant principalement l'exposition "Une montagne de timbres", accompagnée de documents vidéo à la disposition des écoles et des enseignants. Cette exposition, en allemand et en français, a été présentée dans 11 cycles d'orientation et collèges du Haut-Valais et du Bas-Valais, ainsi que dans les trois médiathèques valaisannes (SaintMaurice, Sion et Brigue).

Valais Solidaire a participé à l'organisation des Rencontres de la solidarité, qui ont eu lieu les 4 et 5 avril 2003 à Sierre. Le succès a dépassé les espérances, puisque des centaines de personnes ont participé à une conférence, à une soirée reggae et à la Journée des associations. Le Prix de la solidarité, dont la responsabilité de l'organisation a été assumée par Valais Solidaire, a voulu mettre en lumière les acteurs valaisans de la solidarité et montrer à chacun, notamment aux jeunes, les multiples opportunités d'une action citoyenne à travers de nouvelles formes de solidarité, que ce soit en Valais ou à l'étranger. Les trois projets primés ont représenté, pour leurs responsables, une reconnaissance très utile.

Grégoire Raboud

Président de Valais Solidaire, <gregoire.raboud@netplus.ch>

\section{La mobilisation de ressources financières publiques en faveur des ONG}

Les fédérations jouent un rôle important dans la mobilisation des ressources financières publiques en faveur du développement, aux trois niveaux communal, cantonal et fédéral. Au lieu que chaque organisation s'adresse individuellement à l'entité publique concernée, c'est la fédération, en tant que représentante du mouvement associatif, qui coordonne les relations avec les entités publiques. Il ne s'agit cependant pas d'une relation d'exclusivité ou de monopole, mais plutôt d'une entente informelle qui permet la coordination, qui a l'avantage d'éviter la dispersion et qui donne certaines garanties de qualité déjà évoquées précédemment.

En ce qui concerne les communes, les fédérations incitent celles-ci, à travers un travail d'information et de sensibilisation, à prévoir dans leurs budgets annuels des montants en faveur de la coopération au développement. Il faut dire que beaucoup de communes prennent de leur propre gré des initiatives allant dans ce même sens et s'adressent ensuite aux fédérations pour leur annoncer leur disponibilité. Les fédérations leur soumettent alors des propositions de projets provenant des associations membres que les communes peuvent financer. Elles assurent le suivi, le contrôle et le compte rendu dans les délais.

Sur le plan cantonal, les fédérations sont également des interlocuteurs privilégiés pour canaliser des fonds cantonaux destinés à la coopération internationale ${ }^{6}$. Même si les financements ne passent pas par le truchement de la fédération comme c'est le cas dans le canton du Tessin -, la fédération est néanmoins sollicitée de donner son avis.

6 Cela concerne surtout les cantons de Genève et de Vaud. 
Au niveau de la Confédération, la DDC, qui, conformément à sa politique, prévoit annuellement des financements importants en faveur des ONG, voit un grand intérêt à ce que les fédérations assurent un rôle de «canalisateurs » des fonds, en effectuant un travail de présélection qui soulage le travail de l'administration. En effet, il est pratiquement impossible de traiter individuellement les propositions venant d'ONG de toute la Suisse. Pendant des années, la DDC a approuvé des propositions de projets qui émanaient de membres de la FGC et qui avaient déjà fait l'objet d'une étude préalable auprès de sa commission technique; depuis peu, elle va plus loin en accordant à la FGC un crédit-cadre avec une délégation de pouvoir étendue en ce qui concerne l'approbation des projets. C'est à présent également le cas pour le Canton et la Ville de Genève. Ce même système est introduit pour le financement des projets des associations membres de la FEDEVACO (Fédération vaudoise de coopération). Le système du créditcadre nécessite cependant une capacité d'absorption relativement importante, ce qui fait hésiter certaines fédérations à franchir le pas tout de suite.

\section{FEDEVACO (Fédération vaudoise de coopération), <www.fedevaco.ch>}

La FEDEVACO est née en 1989, sur l'initiative d'une poignée d'organisations désireuses de réunir leurs forces afin de mobiliser les collectivités publiques en faveur de la coopération au développement. Inspirés par l'expérience de la FGC à Genève, les initiateurs vaudois utilisaient des projets concrets pour approcher les collectivités publiques et obtenir leur soutien sous forme de contributions financières.

Quinze ans plus tard, la FEDEVACO regroupe 34 organisations; les contributions financières ont triplé ces cinq dernières années et le travail de lobbying se poursuit à travers des actions concrètes de développement sur le terrain.

Si les premières démarches se faisaient sur une base ponctuelle de financement par projet, dès 2002 la FEDEVACO reçoit un soutien de la Confédération sous forme d'un crédit-cadre, à savoir une enveloppe financière qui est mise à sa disposition sur trois ans et qu'elle gère de façon autonome.

Les avantages de ce crédit-cadre sont multiples: possibilité d'élaborer un budget prévisionnel à moyen terme, plus grande marge de manœuvre dans le financement du "portefeuille» de projets, augmentation des ressources financières pour des petites $0 \mathrm{NG}$, pour n'en citer que les principaux.

C'est, bien sûr, également une responsabilité accrue pour la FEDEVACO puisque la décision et le choix des projets sont désormais de son ressort.

II faut relever que ce type de contrat est pour l'instant l'apanage de la seule Confédération (DDC), le canton et les communes continuant à fonctionner avec des contributions annuelles par projet. La FEDEVACO essaie toutefois d'encourager ces derniers à suivre l'exemple de la DDC, la première étape étant de leur proposer de conserver un rythme annuel de financement, mais de laisser à la FEDEVACO le libre choix de l'attribution finale aux projets.

Patricia Dubois

Coordinatrice de la FEDEVACO, <info@fedevaco.ch>

\section{Un mouvement d'associations qui se "professionnalise"}

En Suisse, nous connaissons un tissu très varié d'associations qui œuvrent en faveur des pays en développement. A part les missions religieuses, qui remontent à plus loin, c'est après la Seconde Guerre mondiale, et plus encore depuis les indépendances d'un grand nombre de pays de l'hémisphère Sud dans les années 1960, que les associations de développement ont commencé à voir le 
jour. Beaucoup de petites associations, laïques ou d'inspiration religieuse, restreintes à un cercle limité d'adhérents et connues sur le seul plan local, œuvrent depuis lors à côté de quelques moyennes et grandes organisations à envergure régionale et nationale ${ }^{7}$.

Or, de nos jours, l'ensemble des organisations, petites ou grandes, est confronté à la difficulté du «métier» de coopérer. Cela est dû à la fois à la complexité des problèmes sociétaux qu'une association veut aider à résoudre, aux tensions du contexte sociopolitique et à l'intrication du cadre institutionnel. En effet, depuis que la coopération existe, les problèmes n'ont pas diminué; malgré des efforts considérables entrepris pour lutter contre ces fléaux, de graves situations de pauvreté, d'exclusion, de sous-alimentation persistent... Cela fait réfléchir! Sur le plan sociopolitique, on observe l'augmentation considérable de situations de conflit, la grande rapidité avec laquelle les changements interviennent, la masse d'informations à disposition, etc. Un autre phénomène, très difficile à gérer pour chaque ONG, est l'accroissement important du nombre d'acteurs qui interviennent. Cela pose des problèmes de rivalité, de concurrence, de reconnaissance, de justification, ce qui finalement augmente les exigences sur le plan de la gestion, de la politique d'information et du travail en réseau. Bref, le «métier» n'est pas facile, d'autant plus qu'il nécessite des moyens adéquats qui ne sont pas toujours disponibles ni faciles à mobiliser. Comment les associations peuvent-elles faire face à ces nouvelles exigences?

Les grandes organisations sont assez bien préparées à affronter ce nouveau contexte. Elles disposent d'une équipe qui a la capacité de travailler sur ces questions, de les analyser et de procéder à des ajustements et des changements. Pour les petites et moyennes organisations, la situation est plus difficile car l'acceptation du nouveau contexte met en quelque sorte en question les modes traditionnels de concevoir la coopération, tels qu'un fort engagement «militant» des membres, un travail basé sur le bénévolat, une estimation minimale des frais administratifs, etc. Toutes ces caractéristiques sont des valeurs très louables qu'il ne faut surtout pas perdre, mais elles ne suffisent plus aujourd'hui pour orienter le travail de coopération. Elles doivent être complétées par des réflexions qui tiennent compte, par exemple

$\checkmark$ des situations complexes dans les pays d'intervention, ce qui nécessite, outre une analyse professionnelle des problèmes à résoudre, une fine analyse des acteurs en présence, des dynamiques de développement déjà existantes, de la situation des partenaires...;

$\checkmark$ des aspects du développement durable, en incluant dans chaque intervention une perspective à long terme ;

$\checkmark$ de la présentation formelle du dossier, qui doit respecter divers critères afin qu'une certaine transparence soit assurée.

Travailler dans cette orientation nécessite des moyens et des compétences professionnelles qui ne sont pas forcément à la portée de toutes les organisations. Les petites et moyennes organisations peuvent ainsi se trouver devant un dilemme sérieux: elles veulent rester petites, car c'est une de leurs forces, et elles veulent continuer à travailler sur une base de bénévolat et avec un fort

7 Pour plus d'informations sur le paysage suisse des ONG, voir la bibliographie et les références de sites Internet à la fin de cet Annuaire. 
engagement de solidarité; si alors elles veulent se former ou se faire aider par des «spécialistes», elles ne disposent pas de ressources suffisantes pour financer des cours ou se procurer les services.

C'est ici que les fédérations entrent en action. Elles ont une fonction de formation et d'appui-conseil en faveur de leurs membres. Elles peuvent soutenir les petites et moyennes associations de manière adaptée à leur situation, répondant à des besoins précis et sur une base de confiance. Elles peuvent aussi aider leurs membres en ce qui concerne la rédaction de documents. Dans la pratique, nous avons pu observer que ces appuis ont débouché sur de réels changements dans des petites organisations, dont la qualité de travail s'est indéniablement améliorée.

\section{Federazione delle ONG della Svizzera italiana, <www.fosit.org>}

La Federazione delle ONG della Svizzera italiana (FOSIT) est née en 1999 avec 49 ONG et en compte aujourd'hui une soixantaine. Un nombre important de petites organisations souhaitait vivement se regrouper. Le besoin d'une certaine professionnalisation des associations a été une des raisons principales de la création de la fédération de la Suisse italienne. En effet, le travail de la plupart des ONG est fondé sur un engagement bénévole, ce qui est à la fois un atout - mais aussi un handicap potentiel. L'atout réside certainement dans le fort engagement personnel des bénévoles, encouragé par la FOSIT. L'handicap est le manque de professionnalisme, qui peut avoir des répercussions sur la qualité. C'est sur ce point que l'équipe des responsables de la FOSIT a beaucoup insisté depuis sa création. Avec le soutien d'un formateur extérieur, la FOSIT a réalisé des séminaires de formation permettant de mettre en commun les différentes expériences des associations membres - et c'est pour donner une continuité à ce travail qualitatif que la FOSIT a été créée. Après quatre années et la réalisation de plus de vingt séminaires de deux jours ou davantage, avec une présence moyenne de dix ONG et plus de vingt personnes par séminaire, on ressent l'importance fondamentale de ce travail de professionnalisation. Les ONG ont pu améliorer leur travail grâce à la consolidation des structures. Sur le plan opérationnel, le niveau de gestion aujourd'hui atteint permet à la plupart des ONG d'accéder aux fonds publics.

Claudio Naiaretti

Secrétaire exécutif de la FOSIT, <fosit@freesurf.ch>

\section{Pouvoir public et ONG : une relation en évolution}

Le fait que les ONG se regroupent en fédérations facilite la relation entre le pouvoir public (aux niveaux fédéral, cantonal et communal) et le monde des ONG. Cela ne concerne pas seulement les relations financières, mais tout type d'échange entre les deux groupes d'acteurs. Que ce soit un canton ou une commune qui veuille développer ses engagements en faveur de la coopération internationale ou que ce soit une ONG qui veuille solliciter un appui de la part du pouvoir public, les fédérations peuvent favoriser l'intermédiation entre les deux groupes. Les expériences montrent que

$\checkmark$ du point de vue de l'intérêt des $O N G$, s'organiser autour d'une fédération permet d'être mieux entendu et pris au sérieux car celle-ci représente un certain nombre d'associations qui ont été choisies selon des règles déontologiques et des critères bien précis, ce qui fournit au pouvoir public une certaine garantie de qualité et de crédibilité;

- du point de vue de l'intérêt du pouvoir public, se trouver face à un interlocuteur organisé dans un secteur bien précis comme celui de la coopération 
internationale, c'est avoir un répondant principal qui représente les diverses associations au lieu de devoir traiter séparément avec chaque ONG. Mais il ne se pose pas seulement la question de l'importance administrative: le pouvoir public, qui doit veiller à l'impartialité, aux intérêts collectifs, aux effets durables de ses interventions, prend un risque moindre en traitant avec une organisation faîtière qui offre un cadre pour veiller au respect de ces exigences. Le pouvoir public peut ainsi se limiter à un rôle de contrôle général et d'orientation politique, et il ne s'occupe pas de l'exécution opérationnelle (selon le principe de la subsidiarité). De plus, le pouvoir public (au niveau communal par exemple) ne dispose pas forcément des compétences internes pour traiter des questions de coopération au développement, comme entre autres étudier les projets qui lui sont soumis pour financement. Pouvoir compter sur une instance spécialisée qui effectue ce travail lui facilite certainement la tâche.

Les fédérations sont en quelque sorte des «facilitateurs» dans la relation entre pouvoir public et monde des ONG. $\mathrm{Si}$, d'une manière générale, cette vision est assez largement partagée, les deux exemples suivants, qui se situent au niveau cantonal, montrent que cette orientation doit être nuancée et que les relations entre pouvoir public et monde associatif peuvent évoluer.

Premier exemple. Dans le canton du Jura, le canton n'avait jusqu'à récemment pas de relations étroites avec la Fédération jurassienne de coopération au développement (FJCD). Cela était notamment dû au fait que le canton a lui-même, par le biais d'un article constitutionnel, un engagement dans la coopération au développement. Le principe, évoqué précédemment, de la subsidiarité vis-à-vis du monde associatif n'est donc pas valable dans ce cas. Il faut cependant préciser que la collaboration entre le canton et la FJCD s'est nettement améliorée et intensifiée ces derniers temps et que le canton appuie aujourd'hui les activités de la FJCD : participation aux coûts du secrétariat, à la Fête de la solidarité, etc.

Second exemple. A Genève, avec l'augmentation des fonds alloués à la coopération internationale ${ }^{8}$, le canton, par le biais du Service de la solidarité internationale, s'occupe également d'actions en faveur de la coopération internationale. Il devient ainsi directement acteur, avec un guichet propre. L'idée n'est pas de se substituer à la Fédération genevoise de coopération (FGC), mais de couvrir d'une manière complémentaire des champs de la solidarité internationale qui n'entrent pas forcément dans les domaines de la FGC. Mais il est clair qu'indirectement, ces fonds entrent en concurrence avec des fonds que la FGC souhaiterait voir augmenter pour les projets qu'elle soutient dans les pays en développement. Le débat en cours à ce sujet est constructif, mais compliqué à cause de la situation difficile des finances cantonales.

8 Depuis 2001, la République et Canton de Genève consacre en principe $0,7 \%$ de son budget annuel de fonctionnement à la solidarité internationale, conformément à la nouvelle Loi (8480) sur le financement de la solidarité internationale. Dans l'exécution de la loi, le canton n'a cependant pas encore pu tenir cet engagement. Malgré le regroupement de toutes les dépenses liées directement ou indirectement à la solidarité internationale, en 2003 le budget s'élève à 10,9 millions, soit $0,17 \%$ du budget total de l'Etat. 


\section{Fédération genevoise de coopération, <www.fgc.ch>}

La Fédération genevoise de coopération (FGC) poursuit plusieurs objectifs, parmi lesquels l'information et le lobbying sur le thème de la solidarité internationale pour défendre, voire augmenter les budgets destinés à la coopération internationale. Ce résultat est le fruit d'un développement institutionnel et d'un partenariat établi dans le long terme et de manière étroite avec les acteurs publics cantonaux et en particulier le canton de Genève, la Ville de Genève, la DDC et les communes genevoises.

Au-delà de sa légitimité politique et sociale, la FGC fournit d'importants services aux pouvoirs publics. Avec un staff technique salarié limité et beaucoup d'heures de bénévolat, elle assure, pour le compte de 47 associations, I'analyse de demandes de financement ainsi que le suivi financier et technique d'un nombre élevé de projets de développement que les pouvoirs publics n'ont pas forcément les moyens d'assurer.

La FGC est donc à la fois un prestataire de services et un interlocuteur des pouvoirs publics pour le financement de projets et un acteur politique critique. Elle a par exemple mené une large campagne en septembre 2003, adressée notamment aux collectivités publiques, sur la nécessité d'augmenter l'aide au développement en posant que la sécurité humaine globale passe par la coopération au développement. Un autre exemple illustrant le rôle politique et social de la FGC sur la scène publique genevoise est le fait qu'elle est citée, dans le Règlement d'application de la récente Loi cantonale sur le financement de la solidarité internationale, "comme un interlocuteur privilégié de l'Etat de Genève" (art. 8) pour la mise en œuvre de sa politique de solidarité internationale. Malgré ce succès et la signature d'accords de financement pluriannuel avec les principaux acteurs publics de la coopération au développement (Etat de Genève, Ville de Genève et DDC), la FGC reste tributaire du bon vouloir de ces derniers et de la situation difficile des finances publiques.

En conclusion, l'expérience genevoise montre que la relation d'une fédération avec les pouvoirs publics ne va pas sans soulever des questions, auxquelles il est toutefois possible de trouver des solutions satisfaisantes grâce à un dialogue développé dans un esprit de partenariat critique. En effet, une fédération cantonale d'associations ne peut se résumer à un guichet de financement de projets, au risque de perdre son âme et son rôle politique et critique au nom de ceux qui souhaitent un autre développement des relations Nord-Sud.

Serge Ghinet

Président de la Commission technique de la FGC

Pour conclure, le travail effectué par les fédérations cantonales nous semble d'une grande utilité pour renforcer le mouvement associatif. Cela vaut avant tout pour les petites et moyennes associations qui fonctionnent encore largement sur la base du bénévolat. Elles ont besoin d'être épaulées car, seules, elles n'ont pas forcément la capacité d'assumer certaines fonctions comme la formation interne, l'information du public, le lobbying et l'entretien des relations avec les entités publiques qui sont des partenaires privilégiés des associations de développement. 